\title{
Faecal chymotrypsin in small for gestational age infants: effects of nucleotides and breast feeding
}

\author{
M Cosgrove, H Losty, H R Jenkins, D P Davies
}

\begin{abstract}
The effect of diet on pancreatic exocrine function, measured by faecal chymotrypsin activity (FCA), was studied longitudinally in three groups of small for gestational age (SGA) infants in the first six months of life. The three groups comprised breastfed infants (group B), those randomly allocated to receive a standard infant formula (group S), or the same formula supplemented with nucleotides (group N). The three groups did not differ in their birthweight or gestational age. Nucleotide supplementation of infant formula improves catchup growth in SGA infants but whether this is due to effects on the gastrointestinal mucosa or the exocrine pancreas is not known.

There were no differences in FCA at study entry but by one month group $B$ had significantly lower values than the other groups, and this was maintained at 2, 4, and 6 months. Groups $\mathrm{N}$ and $\mathrm{S}$ did not differ significantly at any time point.

Nucleotide supplementation of infant formula does not influence pancreatic exocrine function and its effect on growth is unlikely, therefore, to be mediated through the pancreas. This study shows that breast feeding is associated with lower FCA which may be related to the lower protein content of human milk. Reliable interpretation of FCA in young infants requires information about their diet. (Arch Dis Child 1997;76:F201-F202)
\end{abstract}

Keywords: chymotrypsin; small for gestational age; nucleotides; breast feeding; formula feed

Pancreatic exocrine function is influenced by fetal nutrition. Studies in several animal species have shown impaired function and reduced size of the pancreas following experimentally induced $^{1}$ or naturally occurring ${ }^{2}$ intrauterine growth retardation (IUGR). Duodenal juice obtained from small for gestational age (SGA) human infants shows negative correlations between lipase and trypsin activities and the degree of IUGR. ${ }^{3}$ A reliable index of pancreatic exocrine function in the human infant is faecal chymotrypsin activity (FCA): it is non-invasive and relatively inexpensive to measure. ${ }^{4} \mathrm{FCA}$ is significantly lower in SGA infants compared with appropriately grown controls, ${ }^{5}$ and soon after birth strongly correlates with catchup growth over the first six months of life ${ }^{6}$ which provides further evidence for impaired pancreatic exocrine function being associated with intrauterine malnutrition and reduced catchup growth.
Little has been published on how pancreatic exocrine function might be affected by postnatal diet. One cross-sectional study found significantly lower FCA in breastfed infants compared with those fed on standard infant formula milk. ${ }^{7}$ We have already shown that nucleotide supplementation of infant formula improves catchup growth in SGA infants and suggested this may be due to trophic effects of nucleotides on intestinal mucosa damaged by intrauterine malnutrition. ${ }^{8}$ However, this improved growth may also be mediated through a beneficial effect of nucleotides on exocrine function of the pancreas, an endodermal derivative of the upper gastrointestinal tract. To test this hypothesis and to learn more about the effects of postnatal diet on pancreatic exocrine function following prenatal growth retardation, we studied longitudinally the effects of three different diets (human milk, standard infant formula, and nucleotide supplemented formula) on FCA in SGA infants over the first 6 months of life.

\section{Methods}

Infants were recruited to the study over 18 months from four maternity units in South Wales. Inclusion criteria were: gestational age greater than or equal to 37 weeks; birthweight below the 5th centile for length of gestation, allowing for maternal height, birth order, and $\mathrm{sex}^{9}$; and singleton pregnancy. Infants with a major congenital or chromosomal anomaly were excluded.

Recruitment took place within seven days of birth. Infants of mothers deciding not to breast feed were randomly allocated to receive either a standard infant formula (group S) or the same formula supplemented with the five monophosphate nucleotides in concentrations similar to those reported in human milk (group N). Infants who were breastfed were followed up and included as group B if breast feeding continued for at least one month.

Faecal samples were collected at study entry (between 2 and 7 days of age) and then at 1,2, 4 and 6 months of age. Samples were frozen at $-20^{\circ} \mathrm{C}$ within 72 hours of collection and FCA analysed in batches using a commercially available kit (Chymo, Boehringer Mannheim) and an automated spectrophotometer. Chymotrypsin liberates a chromogenic product ( $p$ nitroaniline) from a synthetic tetrapeptide substrate. Each sample was analysed in triplicate and the mean value used for analysis. The assays were performed by staff who were not aware of the infants' diet. FCA values were converted to square roots to normalise their distributions. Differences between the three groups at each time point were analysed by 
Table 1 Characteristics of study infants

\begin{tabular}{|c|c|c|c|}
\hline & $\begin{array}{l}\text { Group } N \\
(n=35)\end{array}$ & $\begin{array}{l}\text { Group } S \\
(n=31)\end{array}$ & $\begin{array}{l}\text { Group B } \\
(n=32)\end{array}$ \\
\hline Mean (SD) gestational age (weeks) & $38.4(1.2)$ & $39.1(1.4)$ & $38.6(1.1)$ \\
\hline Mean (SD) birthweight $(\mathrm{g})$ & $2332(243)$ & $2395(225)$ & $2352(246)$ \\
\hline $\begin{array}{l}\text { Mean (SD) age at introduction of solid } \\
\text { foods (weeks) }\end{array}$ & $14.1(3.9)$ & $12.0(2.6)$ & $14.1(4.1)$ \\
\hline
\end{tabular}

Group N: nucleotide supplemented formula; group S: standard infant formula; group B: breastfed. analysis of variance (ANOVA), and differences between pairs of the three groups using the Bonferroni method.

\section{Results}

Samples were analysed on two or more occasions from 35 infants in group $\mathrm{N}, 31$ infants in group S, and 32 infants in group B. The three groups did not differ significantly in their birthweight, gestational age, nor in the ages when solid food was introduced (table 1). Table 2 gives the number of samples analysed at each time point for each group. The large dropout from group B was largely as a result of infants being changed from breast to formula feeding during the study period. These infants did not differ from those remaining in the study in their initial FCA.

For reasons of clarity figure 1 shows the median FCA before transformation to square roots at each time point for the three groups. After transformation there were no significant differences between the FCA in the three groups at study entry, but by one month group $B$ had significantly lower FCA than the two formula-fed groups (ANOVA $\mathrm{P}=0.006$ ), differences that persisted at 2 months $(\mathrm{P}<0.0001), 4$ months $(\mathrm{P}=0.008)$, and 6 months $(\mathrm{P}=0.025)$. At no time point were there significant differences between groups $\mathrm{N}$ and $\mathrm{S}$.

\section{Discussion}

SGA infants are a heterogenous group but fetal undernutrition is an important cause of their small size. Among infants in this subgroup, early postnatal catchup growth is a recognised and important feature. ${ }^{10}$ However, fetal undernutrition is also associated with impaired structure $^{2}$ and function ${ }^{11}$ of the small intestinal mucosa, and of pancreatic exocrine function, ${ }^{3}$ both of which may limit catchup growth. We have already shown that nucleotide supplementation of infant formula improved catchup growth and suggested that this was likely to be due to trophic effects on the intestinal mucosa. ${ }^{8}$ The possibility that absorption of nucleotides might also enhance pancreatic exocrine function needs to be considered as this might be an alternative explanation for the observed improvement in catchup growth.

Table 2 Numbers of samples collected from infants in three feed groups at different time points

\begin{tabular}{llll}
\hline Age & Group N & Group $S$ & Group $B$ \\
\hline$<1$ week & 33 & 26 & 29 \\
1 month & 30 & 23 & 21 \\
2 months & 22 & 22 & 24 \\
4 months & 19 & 17 & 11 \\
6 months & 14 & 15 & 11 \\
\hline
\end{tabular}

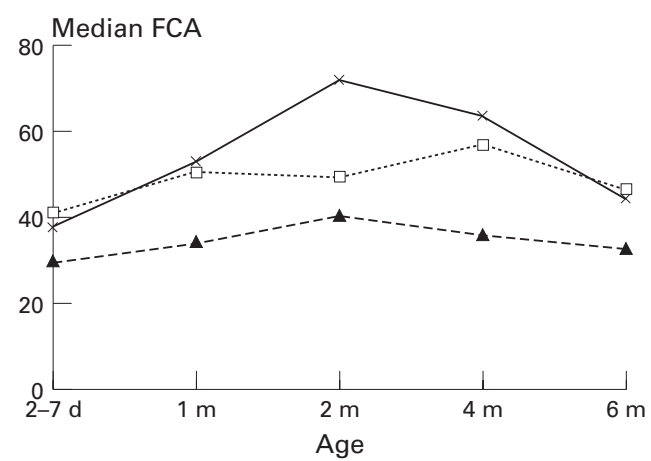

$\begin{aligned} \rightarrow & \text { Standard infant formula (group S) } \\ \cdots-- & \text { Nucleotide supplemented } \\ & \text { formula (group N) } \\ -\_- & \text {Breastfed (group B) }\end{aligned}$

Figure 1 Median FCA (IU/g faeces) in the first 5 months in three different feed groups.

Nucleotide supplementation does not seem to affect pancreatic exocrine function measured by FCA. This indirectly supports our previous hypothesis that the improved catchup growth with nucleotide supplementation is more likely to be mediated through trophic effects on the intestinal mucosa.

Our study confirms that breastfed infants have lower FCA values than those fed with formula, ${ }^{7}$ emphasising the importance of considering diet when interpreting FCA in young infants. The true protein content of human milk is lower than previously thought, ${ }^{12}$ and certainly lower than that in standard infant formula. With the protein content of the diet influencing pancreatic exocrine function, ${ }^{13}$ we speculate this to be the reason for the lower FCA in breastfed infants.

We gratefully acknowledge the technical expertise of Heather Wheatley in performing the assays.

1 Lebenthal E, Nitzan M, Chrzanowski BL, Krantz B. The effect of reduced maternofetal blood flow on the development of fetal pancreatic acinar cells and enzymes. Pediatr ment of fetal pancreatic

2 Xu R-J, Mellor DJ, Birtles MJ, Reynolds GW, Simpson HV. Impact of intrauterine growth retardation on the gastrointestinal tract and the pancreas in newborn pigs. $\mathcal{F}$ Pediatr Gastroenterol Nutr 1994;18:231-40.

3 Boehm G, Bierbach U, Senger H, Jakobsson I, Minali I, Moro G, et al. Activities of lipase and trypsin in duodenal juice of infants small for gestational age. $\mathcal{F}$ Pediatr Gastroenterol Nutr 1991;12:324-7.

4 Brown GA, Sule D, Williams J, Puntis JWL, Booth IW, McNeish AS. Faecal chymotrypsin: a reliable index of exocrine pancreatic function. Arch Dis Child 1988;63:785-9.

5 Kolacek S, Puntis JWL, Lloyd DR, Brown GA, Booth IW. Ontogeny of pancreatic exocrine function. Arch Dis Child 1990;65:178-81.

6 Williams SP, Durbin GM, Morgan MEI, Booth IW. Pancreatic exocrine dysfunction a determinant of catch-up atic exocrine dysfunction a determinant of catch-up
growth in intra-uterine growth retardation. Arch Dis Child growth in intra-uter

7 Ben RA, Dapia L, Bustos D. Fecal chymotrypsin in infants fed different diets. $\mathcal{F}$ Pediatr Gastroenterol Nutr 1993;16:104-5.

8 Cosgrove M, Davies DP, Jenkins HR. Nucleotide supplementation and the growth of term small for gestational age infants. Arch Dis Child 1996;74:F122-5.

9 Tanner JM, Thomson AM. Standards for birthweight at gestation periods from 32 to 42 weeks, allowing for maternal height and weight. Arch Dis Child 1970;45:566-9.

10 Fitzhardinge PM, Inwood S. Long-term growth in smallfor-date children. Acta Paediatrica Scandinavica 1989;349 (Suppl):27-33.

11 Ducker DA, Hughes CA, Warren I, McNeish AS. Neonatal gut function, measured by the one hour blood $\mathrm{D}(+)$ xylose test: influence of gestational age and size. Gut 1980;21:133-6.

12 Räihä NCR. Nutritional proteins in milk and the protein requirement of normal infants. Pediatrics 1985;75:S136-41.

13 Roy AD, Campbell LI, Goldberg DM. Effect of diet on the trypsin and chymotrypsin in the stools of patients with an ileostomy. Gastroenterology 1967;53:584-9. 